\title{
Detailed Field Pattern Is Intrinsic to the Embryonic Mouse Hippocampus Early in Neurogenesis
}

\author{
Shubha Tole and Elizabeth A. Grove \\ Department of Neurobiology, Pharmacology and Physiology, Committees on Developmental Biology and Neurobiology, \\ University of Chicago, Chicago, Illinois 60637
}

There is accumulating evidence that the mammalian cerebral cortex is regionally specified early in neurogenesis. However, the degree and scale of the regional pattern that is intrinsic to different parts of the cortical primordium remains unclear. Here, we show that detailed patterning-the accurate positioning of several areas or fields-is intrinsic to the part of the primordium that generates the hippocampus. A caudomedial portion of the cortical primordium, the site from which the hippocampus arises, was isolated from potential extrinsic patterning cues by maintaining it in explant culture. Explants were prepared at embryonic day (E) 12.5, which is early in hippocampal neurogenesis in the mouse and $3 \mathrm{~d}$ before individual fields are seen by differential gene expression. Allowed to develop for $3 \mathrm{~d}$ in vitro, E12.5 explants upregulate field-specific patterns of gene expression with striking temporal and spatial accuracy. Possi- ble sources of patterning signals intrinsic to the explants were evaluated by removing the cortical hem or presumptive extrahippocampal cortex from the explants. To expose cells to different local positional cues, explant fragments were grafted into ectopic positions in a larger explant. None of these manipulations altered the development of patterned, field-specific gene expression. Finally, explants harvested at E10.5 also upregulate field-specific gene expression, although less robustly. Some hippocampal patterning information is therefore intrinsic to the caudomedial cortical primordium at the time that the first hippocampal neurons are born at E10.5. By E12.5, hippocampal field patterning appears to be well established and resistant to the manipulation of several potential intrinsic cues.

Key words: mouse; telencephalon; cerebral cortex; hippocampus; hippocampal fields; area patterning; explant culture
Classic models of early cerebral cortical patterning suggest two distinct primary mechanisms for the division of cortex into cytoarchitectonic areas (Rakic, 1988; O'Leary, 1989). In one model, area patterning arises relatively late in corticogenesis, initiated by innervation from the thalamus (O'Leary, 1989). In the second, areas develop from a protomap that is intrinsic to the cortical primordium early in neurogenesis (Rakic, 1988). Accumulating evidence supports the second alternative. First, in macaque monkeys, in which different stages of cortical development are well separated in time, region-specific gene expression is seen in the cortex before thalamic innervation (Donoghue and Rakic, 1999a,b). Second, in mice deficient for Mash1 or Gbx2, the thalamus fails to innervate the cortex, yet region-specific molecular markers are upregulated in cortex in a patterned manner (Miyashita-Lin et al., 1999; Nakagawa et al., 1999). Third, when directly tested in culture or grafting experiments, different regions of cortical primordium reveal that they are already specified to express different molecular markers. These markers include the $\mathrm{H}-2 \mathrm{Z} 1$ transgene, expressed in postnatal somatosensory cor-

\footnotetext{
Received June 9, 2000; revised Nov. 27, 2000; accepted Dec. 5, 2000.

This work was supported by grants from the National Institutes of Health, the March of Dimes, and the Brain Research Foundation (E.A.G.), and by a fellowship from the Committee on Cancer Biology, University of Chicago (S.T). We thank J. Boulter, G. Lemke, and S. Nakanishi for gifts of plasmid DNA, Tim Sanders and Cliff Ragsdale for their two-color in situ hybridization and BrdU labeling procedures, Stavroula Assimacopoulos and Eun Paik for technical assistance, and the staff of the BSD Audio-Visual Communications, University of Chicago, for their help with figure preparation.

Correspondence should be addressed to Elizabeth A. Grove, Department of Neurobiology, Pharmacology, and Physiology, 947 East 58th Street, University of Chicago, Chicago, IL 60637. E-mail: egrove@drugs.bsd.uchicago.edu.

Dr. Tole's present address: Department of Biological Sciences, Tata Institute of Fundamental Research, Mumbai 400005, India. E-mail: stole@tifr.res.in. Copyright (C) 2001 Society for Neuroscience $0270-6474 / 01 / 211580-10 \$ 15.00 / 0$
}

tex; LAMP, a limbic cortex marker; latexin, expressed in lateral cortex; and Otx2, a marker of prospective visual cortex (Arimatsu et al., 1992, 1999; Ferri and Levitt, 1993; Cohen-Tannoudji et al., 1994; Eagleson et al., 1997; Levitt et al., 1997; Nothias et al., 1998; Gitton et al., 1999).

A regional pattern is therefore intrinsic to the cortical primordium, but the scale and detail of this pattern remain unclear. That is, the primordium might be just broadly regionalized, with precise area boundaries established only near or after birth, perhaps by thalamic or other extrinsic afferents. Alternatively, a more complete area pattern might be present, a pattern in which areas are already well delimited and positioned accurately with respect to one another. Supporting the latter alternative, the H-2Z1 transgene is upregulated in explants of neocortical primordium in a clearly bounded territory, suggesting that presumptive somatosensory cortex, which forms the template for $\mathrm{H}-2 \mathrm{Z} 1$ expression, is well delimited from an early stage (Gitton et al., 1999).

We have used the embryonic mouse hippocampus as a model system for determining the detail of intrinsic patterning in a part of the cortical primordium. The hippocampus is well suited to this purpose because it is divided into a small number of major fields, each of which can be identified by specific patterns of gene expression (Tole et al., 1997, 2000a,b; Lee et al., 2000b), and it develops in a restricted, readily identified region (see Fig. 1 $a, c, d$ ). In this study, the embryonic hippocampus was tested for intrinsic field pattern by isolating the entire hippocampal primordium from extrinsic patterning cues and maintaining it in explant culture. Explants were prepared at different stages of hippocampal neurogenesis and maintained until they reached a stage comparable with the age at which hippocampal fields begin to differentiate in vivo (Tole et al., 1997). The explants were then assayed 

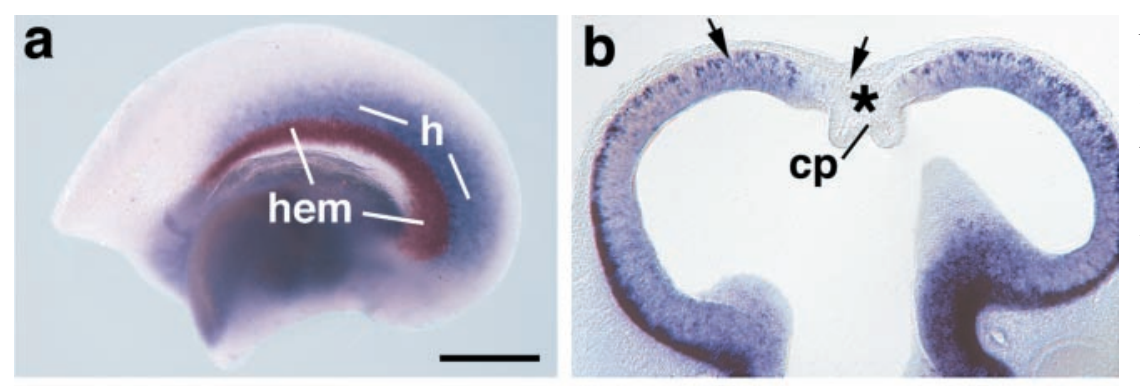

Figure 1. Position of the hippocampal primordium at E10.5 and E12.5. $a, c, d$, E12.5 telencephalic hemisphere processed for two-color in situ hybridization $(a)$, viewed from the medial face, rostral to the left. Within the medial wall of the telencephalon, the curving cortical hem is marked by Wnt3a expression (brown). Dorsal to it, the hippocampal primordium $(h)$, also a curving, longitudinal band, expresses EphB1 (blue). c, The same structures are shown in a coronal section through a similarly processed hemisphere. Again, the cortical hem is marked by Wnt3a expression (brown), and the hippocampal primordium is marked by EphB1 expression (blue). $d$, In a coronal section at a similar rostrocaudal level, differentiating neurons are marked by class III $\beta$-tubulin mRNA expression (blue). The dense layer of differentiating neurons is tapered in the cortical hem (white arrow), broader in the hippocampal primordium $(h)$, and narrowed slightly again at the presumed transition to extrahippocampal cortex (asterisk). Numbered arrows in $c$ indicate the positions at which cuts were made in the medial wall to generate the E12.5 explants used in this study. Initial explants contained the entire dorsal to ventral extent of the medial wall between 1 and 4 . To remove the cortical hem, a further cut was made at 3 . To remove presumptive extrahippocampal cortex, a further cut was made at 2. cpe, Choroid plexus epithelium. $b$, Coronal section through the dorsomedial telencephalon at E10.5, processed for class III $\beta$-tubulin. At E10.5, the dorsal midline of the telencephalon has just begun to invaginate to form the medial walls of each hemisphere (asterisk). The choroid plaque $(c p)$ is at the midline. No hippocampal primordium is discernible, but is likely to be close to the midline. Arrows indicate the cuts that were made to generate E10.5 explants for this study. Scale bars: $a, 470 \mu \mathrm{m} ; b, 166 \mu \mathrm{m} ; c, d, 120 \mu \mathrm{m}$.

to determine if they develop molecular features of hippocampal fields and how the organization of the in vitro field pattern compares with that seen in vivo.

\section{MATERIALS AND METHODS}

\section{Animals}

Timed-pregnant female CD-1 mice were obtained from the University of Chicago Transgenic Facility and killed by $\mathrm{CO}_{2}$ inhalation. Noon of the day on which a vaginal plug was noted was termed embryonic day (E) 0.5 .

\section{Preparation of explants}

Because specific growth factors can regulate expression of regional cortical molecular markers (Levitt et al., 1997), explant culture conditions were designed so that no undefined growth factors were available to the explants from extrinsic sources to provide potential field specification signals. The overlying meninges and other mesenchymal tissues were removed, and explants were maintained in serum-free medium consisting of DMEM with B27 supplement (Life Technologies, Gaithersburg, MD).

\section{E12.5 explants}

E12.5 embryos were dissected in L15 medium (Life Technologies) to isolate the telencephalon. To separate neural tissue from mesenchyme, each telencephalon was treated with $1 \mu \mathrm{g} / \mathrm{ml}$ protease XIV (Sigma, St. Louis, MO), for $3 \mathrm{~min}$, after which the protease was inactivated in L15 containing $10 \%$ calf serum. Mesenchyme was removed with fine forceps. Most of the medial wall of the telencephalic hemisphere was dissected out (Fig. 1c; see Fig. 4), leaving behind only the most rostral portion, which is not part of the presumptive hippocampus. Explants were cultured on Millipore (Bedford, MA) filter inserts for $3 \mathrm{~d}$. At the end of the culture period, explants were fixed in $4 \%$ paraformaldehyde and processed for in situ hybridization.

\section{E12.5 explant manipulations}

Cortical hem removal. The extreme medial edge of the cortical neuroepithelium, the cortical hem, was removed from E12.5 explants as indicated in Figures $1 c$ and $7 a$. Within the E12.5 medial telencephalic wall, the cortical hem could be identified as a thinner, more translucent tissue than adjacent cortex. Removal of the hem was confirmed by processing explants with in situ hybridization to show Wnt $2 b$ expression, which distinguishes the cortical hem from E11.5 to birth.

Removal of extrahippocampal cortex. The E12.5 explants as initially prepared contained not only the hippocampal primordium and cortical hem but also areas of presumptive medial neocortex adjacent to the hippocampal primordium, probably including parts of cingulate and retrosplenial cortex. Unlike the cortical hem, this neighboring medial cortex has not been found as yet to express signaling proteins that might act to pattern the hippocampus. Nonetheless, in principle, this tissue might provide such cues. To test this possibility, we prepared E12.5 explants in which presumptive extrahippocampal cortex was dissected away (Fig. 1c). The approximate boundary between the hippocampal primordium and extrahippocampal cortex (Fig. 1c, cut 2) was determined on the basis of both morphological and gene expression data. For example, by E12.5, expression of the ephrin receptor EphB1 fills the likely hippocampal primordium but does not extend into adjacent medial cortex (Tole et al., 2000a,b) (Fig. 1a,c). The outline and size of the hippocampal primordium was therefore determined in E12.5 telencephalic hemispheres processed to show expression of EphB1 (Fig. 1a,c). These measurements, together with the slight morphological bulge of the hippocampal primordium in fresh tissue and previous illustrations of the embryonic rodent hippocampal-extrahippocampal transition (Altman and Bayer, 1990), were then used to guide subdissection. To further ensure that all parahippocampal cortical tissue was removed, we prepared additional, very reduced explants in which a cut was made partway between cuts 2 and 3 in Figure $1 c$, thus removing not only medial neocortical tissue, but also the subicular fields and part of CA1.

Ectopic grafting. In a final manipulation, small portions of the E12.5 medial telencephalic wall were grafted into ectopic positions within a larger explant as shown in Figure 7e. Subdissected, wedge-shaped portions of the E12.5 medial wall, $\sim 0.4 \times 0.3 \mathrm{~mm}$, were inserted into a wedge-shaped opening cut into the lateral edge of a larger medial wall explant, $\sim 2.5 \times 1.2 \mathrm{~mm}$.

\section{E10.5 explants}

E10.5 explants were prepared by cutting down the midline of the telencephalon and dissecting out the most dorsomedial portion of the embryonic cortex (Fig. 1b). The same culture conditions were used as for E12.5 explants. After $5 \mathrm{~d}$ in vitro (DIV), E10.5 explants were fixed and processed the same as for E12.5 explants.

\section{E15.5 tissue}

Patterns of field- and region-specific gene expression were evaluated in E10.5 and E12.5 explants after 5 or 3 DIV, respectively, and compared with the patterns seen normally at E15.5 in vivo. To visualize gene expression patterns at E15.5 in a preparation that would match the cultured explants, the medial telencephalic wall was dissected out at E15.5, placed onto a Millipore filter insert, allowed to flatten, then fixed and processed for in situ hybridization (see Fig. 4). 


\section{In situ hybridization}

Single- or two-color in situ hybridization was performed as described previously (Tole et al., 1997; Grove et al., 1998). Explant cultures were processed as whole mounts to detect regional expression of $S C I P, K A 1$, NK3, Steel, and other selected genes.

\section{5-bromo-2'-deoxyuridine labeling and detection}

In vitro. Neurons generated by cell proliferation in explant culture were labeled by applying 5-bromo-2'-deoxyuridine (BrdU) $(3 \mu \mathrm{g} / \mathrm{ml}$ final concentration) to E12.5 explants on days 1 and 2 of the $3 \mathrm{~d}$ culture period. At the end of the culture period, explants were fixed in $4 \%$ paraformaldehyde in PBS and sectioned on a cryostat at $10 \mu \mathrm{m}$. Explants were first processed for in situ hybridization to detect markers of differentiating neurons, such as GAP43 or class III $\beta$-tubulin mRNA expression, then processed for BrdU with peroxidase immunohistochemistry as described previously (Grove et al., 1998).

Various control explants were prepared to test the specificity of the BrdU staining. These control explants were (1) untreated with BrdU, (2) treated with BrdU on day 3 only of the $3 \mathrm{~d}$ culture period so that fewer cells would be labeled, or (3) treated on days $6-10$ of a $10 \mathrm{~d}$ culture period when few or no new neurons should have been generated. Control explants that had not been treated with BrdU were entirely free of staining. As expected, BrdU-labeled cells were much less numerous in explants treated with BrdU on day 3 of the culture period. Finally, when explants were treated on days $6-10$ of a $10 \mathrm{~d}$ culture period (roughly equivalent to E18.5 to postnatal day 2), several cells in the explants were BrdU-labeled, but none coexpressed GAP43 or class III $\beta$-tubulin, suggesting that these cells were not neurons (see Fig. $3 c$ ).

In vivo. A stock of $10 \mathrm{mg} / \mathrm{ml} \mathrm{BrdU}$ was used for in vivo injections at 50 $\mathrm{mg} / \mathrm{kg}$. Pregnant dams were given a single intraperitoneal injection at E10.5, E12.5, or E14.5. Embryos were harvested at E16.5 and processed for BrdU detection as previously described (Grove et al., 1998), with one modification. BrdU was detected with a two-step immunohistochemical procedure, first incubating the sections with an FITC-conjugated antiBrdU antibody (mouse IgG, clone B44; Becton Dickinson, Mountain View, CA), followed by an incubation with an alkaline phosphatase (AP)-conjugated anti-fluorescein antibody (Fab fragments, sheep IgG; Roche Diagnostics, Indianapolis, IN). Sections were then processed to show AP activity, as for the last step of the in situ hybridization procedure. This method is more sensitive than the standard peroxidase immunohistochemical procedure used for BrdU detection and results in the detection of many more labeled cells. Note, for example, the high density of labeling seen in brains labeled with BrdU at E14.5 and analyzed at E16.5 (see Fig. 2c). Control sections obtained from brains that were not exposed to BrdU were entirely free of staining.

\section{RESULTS}

\section{Explant harvest times represent early stages in hippocampal neurogenesis}

The hippocampus develops in the dorsomedial telencephalon, which by approximately E11 in the mouse begins to invaginate to form the medial walls of the two telencephalic hemispheres. Overt hippocampal field patterning emerges in the medial telencephalic wall at E14.5-E15.5 when hippocampal fields begin to differentiate with respect to a panel of gene expression markers (Tole et al., 1997; Grove and Tole, 1999; Lee et al., 2000b). The primary purpose of this study was to determine whether hippocampal field patterning is intrinsic to the dorsomedial telencephalon at earlier stages of neurogenesis, before overt hippocampal fields are seen. E10.5 and E12.5 were selected as appropriate ages provisionally on the basis of previous reports (Angevine, 1965; Caviness, 1973; Stanfield and Cowan, 1979) that neurogenesis begins in the mouse hippocampus at approximately E10.5 and does not peak for pyramidal neurons until several days later. Dentate gyrus neurons begin to be generated at about the same time as CA pyramidal cells, although most dentate neurogenesis occurs in late embryonic and early postnatal life (Angevine, 1965; Caviness, 1973; Stanfield and Cowan, 1979; Altman and Bayer, 1990).
BrdU labeling was used to confirm that E10.5 and E12.5 represent early stages in hippocampal neurogenesis in the CD-1 strain of mice used in this study. To label cells that became postmitotic at different ages, we injected pregnant mice once with BrdU at E10.5, E12.5, or E14.5. Embryos were harvested at E16.5, and BrdU incorporation was analyzed. After an injection of BrdU at E10.5 and harvest at E16.5, labeled cells are extremely sparse in the hippocampal cortical plate-the developing pyramidal cell layer - and in the region of the dentate gyrus (Fig. 2a). After an injection at E12.5, more labeled cells appear in the hippocampus, but BrdU-positive cells in the pyramidal cell layer remain relatively sparse (Fig. 2b). By contrast, after a BrdU injection at E14.5, the hippocampus contains numerous labeled cells (Fig. 2c). Although mouse E14.5 represents a relatively early stage in rodent hippocampal gliogenesis (Altman and Bayer, 1990), some of these BrdU-positive cells may be early generated glia or glial progenitor cells; for example, those in the fimbria, which contains no neurons (Fig. 2c). However, most of the increased cell labeling in the hippocampal cortical plate appears likely to reflect an increase in the production of neurons. Notably, few labeled cells appear in the dentate gyrus region or developing stratum radiatum, although these regions will contain glia as well as neurons (Fig. 2c).

Cortical neurons begin to express class III $\beta$-tubulin mRNA shortly after they become postmitotic and as they are migrating through the intermediate zone to the developing cortical plate. Expression of class III $\beta$-tubulin was therefore also used to evaluate general hippocampal development at E10.5 and E12.5. At E10.5, class III $\beta$-tubulin expression reveals few differentiating neurons in the most dorsomedial telencephalon, the future site of the hippocampal primordium (Fig. 1b). By E12.5, this region has invaginated to form the medial walls of the two telencephalic hemispheres, and the hippocampal primordium can be at least roughly outlined (Fig. $1 c, d$ ). Class III $\beta$-tubulin-positive cells appear in a dense band, but this band of differentiating neurons remains thin compared with the proliferative ventricular zone, which is primarily unlabeled (Fig. 1d).

In summary, at E10.5 and E12.5, when explants were harvested for this study, the portion of the dorsomedial telencephalon that generates the hippocampus is composed primarily of proliferating cells and relatively few differentiating neurons. Testing the patterning ability of explants prepared at these ages therefore tests the intrinsic patterning of the hippocampal primordium at the earliest stages of its development.

\section{Proliferation proceeds in explant culture and newborn cells differentiate as neurons}

The general health and development of the explant cultures was tested by assaying for continued neuronal proliferation and differentiation in vitro (Fig. 3). We prepared explants of the caudal two-thirds of the medial wall of the telencephalon (Fig. 4). Proliferating cells were labeled in E12.5 explants by adding BrdU on days 1 and 2 of culture. At the end of the $3 \mathrm{~d}$ culture period, explants were double-labeled for BrdU and a selected marker of differentiating neurons. Numerous BrdU-labeled neurons were found to express the general neuronal markers GAP-43 (Fig. 3b) or class III $\beta$-tubulin, showing that substantial numbers of neurons are born and differentiate in vitro. Most differentiating neurons collect in a layer toward the pial surface of the explant, suggesting that many neurons born in the explant migrate away from the ventricular surface, as they would in vivo, to form an in vitro equivalent of the cortical plate (Fig. $3 a$, asterisk). Nonethe- 

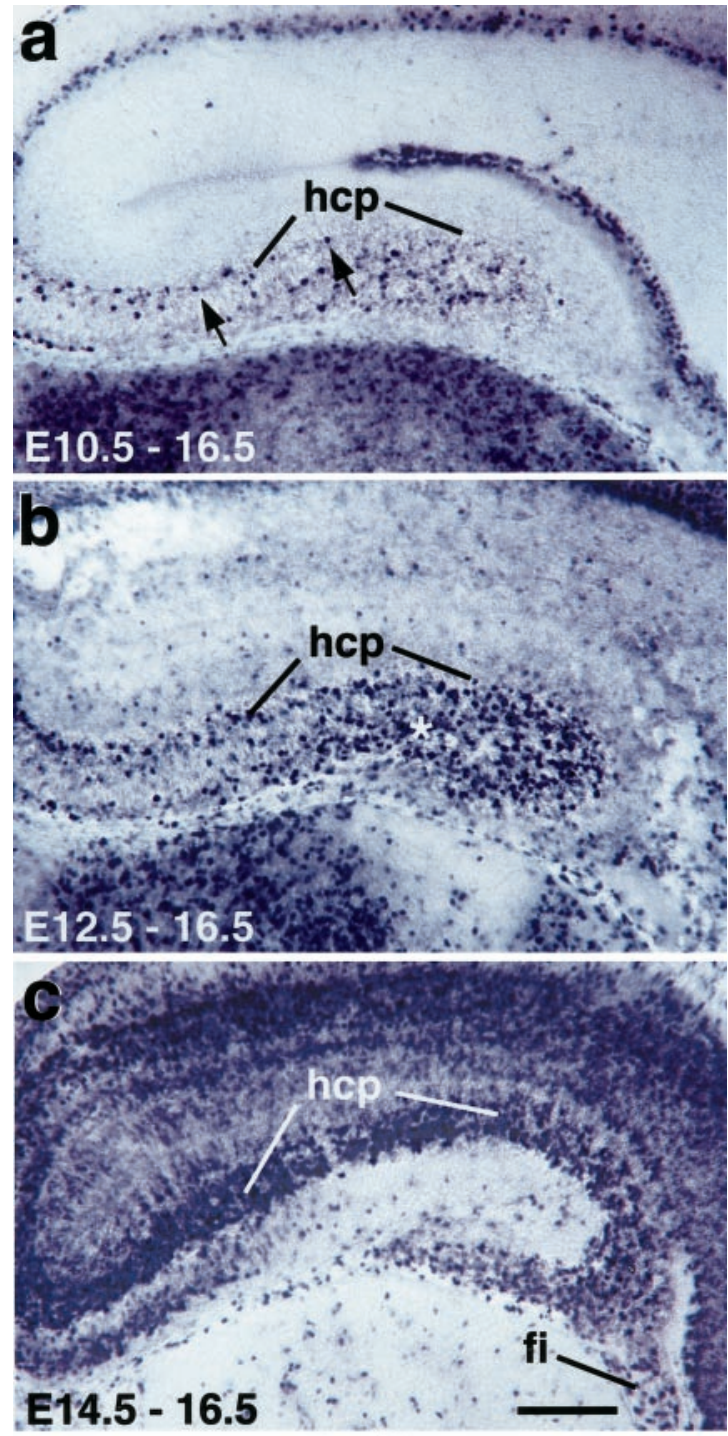

BrdU

Figure 2. Explant harvest times at E10.5 and E12.5 represent early stages in hippocampal neurogenesis. $a-c$, Coronal sections of E16.5 hippocampus, processed to detect BrdU-immunoreactive neurons labeled after BrdU injections at E10.5 $(a)$, E12.5 $(b)$, or E14.5 $(c)$. Strongly BrdU-immunoreactive (BrdU+, dark blue) cells are those that became postmitotic shortly after the BrdU injection. $a$, After a BrdU injection at $\mathrm{E} 10.5, \mathrm{BrdU}+$ cells are sparse in the neocortex and hippocampal cortical plate ( $h c p$, the future pyramidal cell layer), indicating that few neocortical or hippocampal neurons are born at E10.5. Arrows indicate two BrdU+ cells in the hcp. $b$, More BrdU+ cells appear in the hippocampus after an injection at E12.5, but still relatively few in the hippocampal cortical plate. Asterisk indicates labeled cells outside the hippocampal cortical plate. c, A dramatic increase occurs in BrdU+ cells in both the neocortex and the hippocampus after an injection at E14.5. fi, Fimbria. Scale bar, $200 \mu \mathrm{m}$.

less, differentiating neurons also appear in ectopic positions not far from the ventricular surface of the explant (Fig. 3a, arrowheads), suggesting that radial migration is not completely preserved in the explants, as noted previously for slice cultures (Tole et al., 1997).

\section{E12.5 explants develop a field pattern revealed by gene expression}

BrdU-labeled neurons were also found to express SCIP (Fig. 3d), a POU-domain gene that is expressed in the pyramidal neurons of
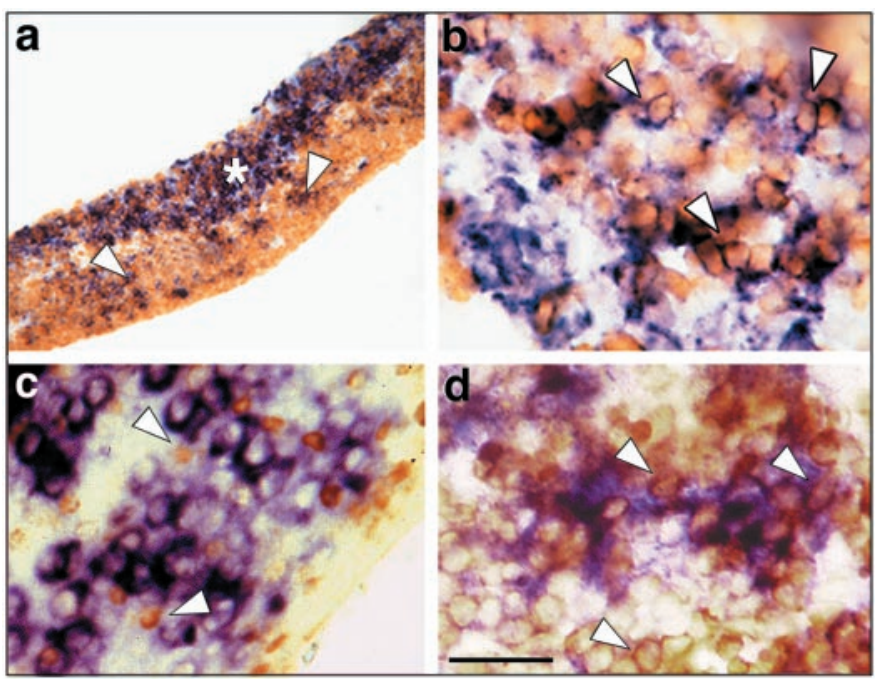

Figure 3. Cells are born in E12.5 explants and upregulate neuronal and regional markers in vitro. $a, b, d$, Cryostat sections through E12.5 explants maintained for $3 \mathrm{DIV}$, processed with in situ hybridization to show GAP43 $(a, b)$, or SCIP $(d)$ expression, followed by BrdU immunohistochemistry. After exposure to BrdU on days 1 and 2 of the culture period, explants display $\mathrm{BrdU}$ immunostaining $(a, b, d$, light to medium brown). Numerous cells express the neuronal marker GAP43 ( $a, b$, blue cytoplasm staining), indicating that cells that divided in vitro gave rise to postmitotic neurons. Many GAP43-expressing neurons appear to have migrated toward the pial surface of the explant to collect in a developing cortical plate ( $a$, asterisk), although other neurons have failed to migrate completely ( $a$, arrowheads). Cells double-labeled for BrdU and the regional marker SCIP ( $d$, purple) indicate that cells born during the culture period acquire a regionally differentiated phenotype. Arrowheads $(b, d)$ indicate doublelabeled cells. $c$, Consistent with a slowing of neurogenesis as the culture period progresses, comparatively few cells were labeled by exposure to BrdU on days $6-10$ of a $10 \mathrm{~d}$ culture period, and these cells did not coexpress GAP43 (purple). Arrowheads indicate two BrdU+/GAP43cells. Numerous GAP43+/BrdU - cells are also evident in the explant ( purple cytoplasm staining only). Scale bars: $a, 160 \mu \mathrm{m} ; b, c, d, 40 \mu \mathrm{m}$.

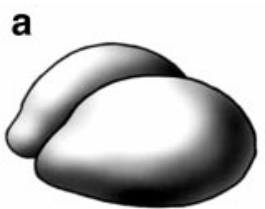

Whole telencephalon

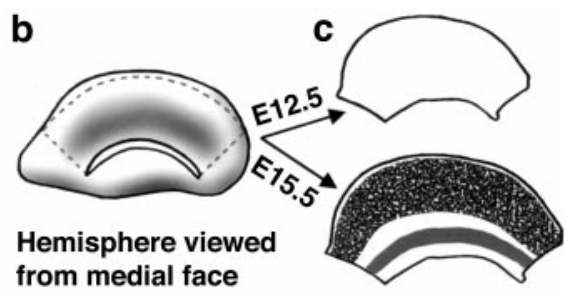

Medial wall explants
Figure 4. Preparation of explants. $a, b$, Individual telencephalic hemispheres were dissected from E12.5 or E15.5 mouse embryo brains (left, rostral; top, dorsal). For an initial set of explants, most of the medial wall of the telencephalon was dissected away ( $b$, broken line $)$ and placed on a filter. c, Bottom, E15.5 medial wall tissue processed immediately for in situ hybridization displays field-specific gene expression patterns. The hippocampal fields appear as curving, longitudinal bands. Top, E12.5 explants processed immediately do not show these field-specific gene expression patterns.

CA1 but not other hippocampal fields beginning at E15.5 (Tole et al., 1997). Thus, neurons born during the culture period also go on to upregulate expression of a characteristically regionally expressed gene. Is gene expression upregulated in field-specific patterns in the explants as it is in vivo?

To determine whether a detailed field pattern develops, the explants were processed as whole mounts for in situ hybridization 
Figure 5. Regional patterning is intrinsic to medial telencephalic explants at E12.5. $a-l$, Explants of the medial wall of the telencephalon processed with in situ hybridization to show expression of the regionspecific markers SCIP, KA1, NK3, and Steel. c, f, i, l, In E15.5 tissue, marker expression appears in concentric curving bands that correspond to the embryonic hippocampal fields. SCIP is expressed in a broad outer band in CA1 and adjacent extrahippocampal cortex (c), KA1 is expressed close to the medial edge of the tissue in CA3 $(f)$, and NK3 expression nests between the $S C I P$ and $K A 1$ domains in contiguous parts of CA1 and CA3 $(i)$. The dentate gyrus is marked by a dark band of Steel expression near the medial edge of the tissue $(l$, arrow). $a, d, g, j$, No marker expression is seen in E12.5 explants processed immediately. $b, e, h, k$, By contrast, E12.5 (3DIV) explants show patterns of marker expression that closely match those in E15.5 tissue. SCIP is expressed in a broad outer band $(b)$, $K A 1$ is expressed near the medial edge $(e)$, and an $N K 3$ expressing band is intermediate between $S C I P$ and $K A 1(h)$. A narrow, medial band of Steel expression ( $k$, arrow) indicates the development of dentate gyrus cells. Scale bars: $a, d, g, j, 0.75 \mathrm{~mm} ; b, e, h, k$, $0.95 \mathrm{~mm} ; c, f, i, l, 1.0 \mathrm{~mm}$.

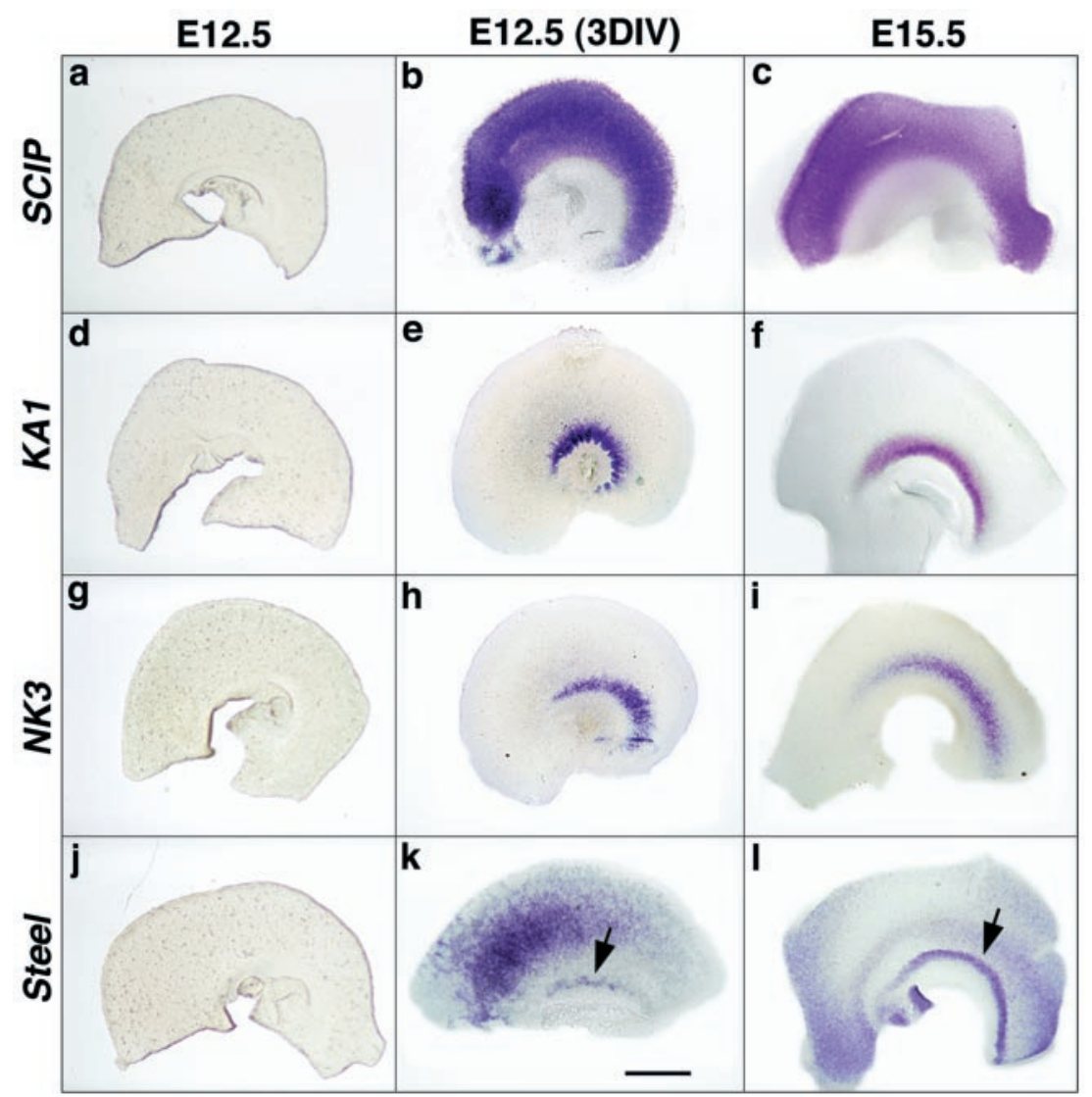

to detect regional expression of several genes, three of which, in vivo, are permanently expressed in particular hippocampal fields. From E14.5 to E15.5 into adulthood, $K A 1$, which encodes a glutamate receptor subunit, and $S C I P$, a POU-domain gene, are expressed in the pyramidal neurons of CA3 and CA1, respectively (Wisden and Seeburg, 1993; Frantz et al., 1994; Tole et al., 1997, 2000a,b; Lee et al., 2000b). Steel, which encodes the c-kit ligand, has a variable and dynamic pattern of expression in the hippocampus but is expressed consistently in the granule cells of the dentate gyrus from E15.5 into adulthood (Motro et al., 1991; Lee et al., 2000b; Tole et al., 2000a,b). NK3 encodes a neuromedin K receptor (Shigemoto et al., 1990) and is transiently expressed in the embryo and neonate in contiguous parts of CA1 and CA3 (Tole et al., 2000a,b).

Patterns of gene expression were compared among E12.5 explants maintained for 0 or 3 DIV and medial telencephalic walls harvested at E15.5. In the E15.5 tissue, expression of the four genes appears in concentric curving bands that correspond to the embryonic hippocampal fields. SCIP is expressed in a broad outer band in CA1 and adjacent extrahippocampal cortex (Fig. 5c), $K A 1$ is expressed close to the medial edge in CA3 (Fig. $5 f$ ), and $N K 3$ expression nests between $S C I P$ and $K A 1$ in adjacent parts of CA1 and CA3 (Fig. 5i). The dentate gyrus is marked by a dark band of Steel expression near the medial edge of the explant (Fig. $5 l$ ). As expected, explants processed immediately at E12.5 reveal no detectable expression of any of the four genes (Fig. $5 a, d, g, j$ ). By contrast, after 3 DIV, explants have upregulated the expression of each gene in a localized and robust manner that compares well with the normal regional pattern at E15.5. SCIP is expressed in a broad outer band (Fig. $5 b$ ), KA1 is expressed close to the medial edge (Fig. 5e), and the $N K 3$ expressing band is interme- diate between $S C I P$ and $K A 1$ (Fig. $5 h$ ). Additionally, the bands of $N K 3$ and $K A 1$ expression in E12.5 (3 DIV) explants are remarkably close in absolute width $(200-300 \mu \mathrm{m})$ to those in E15.5 tissue (Fig 5, compare e/f and $h / i$ ). Finally, a medial band of Steel expression indicates the development of dentate gyrus cells in the E12.5 (3 DIV) explants (Fig. 5k).

Additional genes are expressed transiently in characteristic regional patterns in the embryonic hippocampus, including those that encode the activin receptor, ActRII; the ephrin receptor, EphB1; and the glutamate receptor, iGluR7 (Tole et al., 2000a,b). Expression of these three genes was examined in a small number of explants with similar results: patterned gene expression seen in E12.5 (3 DIV) closely matches that seen in E15.5 tissue (data not shown).

To assess the spatial accuracy of the field pattern further, explants were assayed for expression of two genes at once. Explants prepared for these experiments included the extreme medial edge of the cortical neuroepithelium, the Wnt-rich cortical hem (Grove et al., 1998). Wnt $2 b$ expression was therefore used to mark the cortical hem and provide a medial landmark in the explants. In E15.5 tissue, the band of strong KA1 expression, marking all of CA3, is close to the Wnt-expressing cortical hem (Fig. $6 d, h$ ). A wider gap appears between the cortical hem and the band of NK3 expression, which marks only a part of CA3 as well as a contiguous part of CA1 (Fig. $6 b, f)$. The same relative positioning of $W n t 2 b, N K 3$, and $K A 1$ expression appears in E12.5 (3 DIV) explants (Fig. 6, compare $a / b, c / d$, e/f, and $g / h$ ). Additional two-color in situ hybridization confirmed that the domains of $K A 1, N K 3$, and SCIP expression are nested in the explants as they are in vivo (data not shown).

Virtually all E12.5 (3 DIV) explants showed similar behavior 
E12.5 (3DIV)

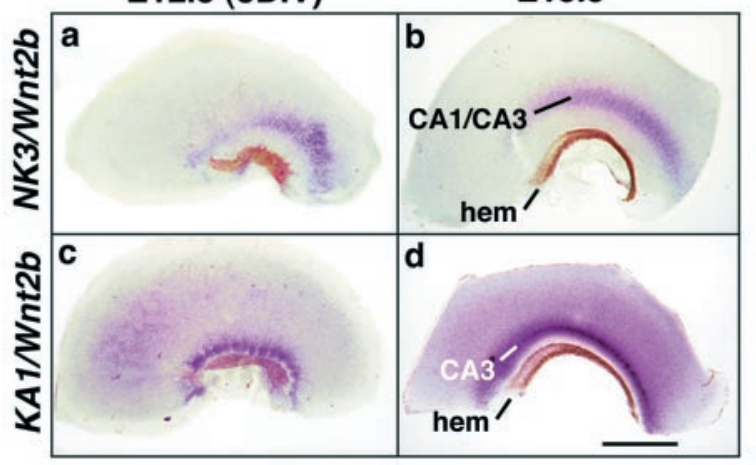

E12.5 (3DIV)

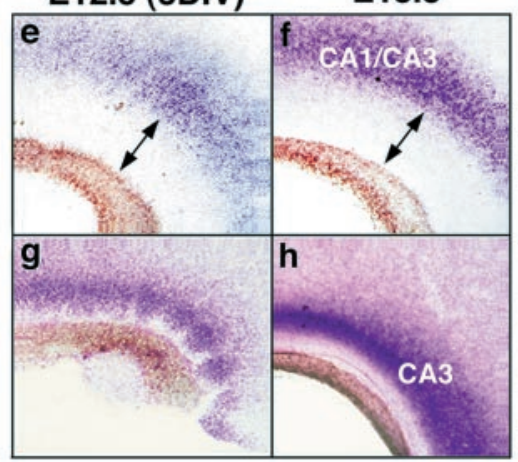

Figure 6. Upregulation of regional markers in E12.5 explants is positionally accurate. $a-h$, Explants of the medial wall of the telencephalon processed with two-color in situ hybridization to show expression of the region-specific markers $W n t 2 b, K A 1$, and $N K 3$. Wnt2b expression marks the Wnt-rich cortical hem in each explant $(b, d$, hem $)$, providing a medial landmark. $K A 1$ expression marks CA3 $(d, h)$, whereas $N K 3$ expression marks cells on either side of the CA1/ CA3 transition $(b, f)$. Thus, in E15.5 tissue, the curving band of $K A 1$ expression is closer to the Wnt $2 b$-expressing hem than is the band of $N K 3$ expression (compare $b$ and $d, f$ and $h$ ). The same relative positioning of bands of $W n t 2 b, N K 3$, and $K A 1$ expression appears in E12.5 (3DIV) explants (compare $a$ and $c, e$ and $g$ ). Even the absolute distances between bands appear similar in E15.5 tissue and E12.5 (3DIV) explants (e,f, arrows). Scale bars: $a, c, 0.95 \mathrm{~mm} ; b, d, 1.0 \mathrm{~mm} ; e-h, 0.27 \mathrm{~mm}$.

Table 1. Regional markers upregulated in E12.5 (3DIV) explants

\begin{tabular}{llll} 
& \multicolumn{2}{l}{$\#$ of explants showing } & \\
\cline { 2 - 4 } Marker & $\begin{array}{l}\text { Correct regional } \\
\text { expression }\end{array}$ & $\begin{array}{l}\text { Incorrect regional } \\
\text { expression }\end{array}$ & No expression \\
\hline SCIP & $25(96 \%)$ & 1 & \\
KA1 & $56(93 \%)$ & & 4 \\
NK3 & $46(85 \%)$ & 3 & 5 \\
Steel & $40(98 \%)$ & & 1 \\
\hline
\end{tabular}

with respect to regional marker expression (Table 1, data pooled from 10 batches of explants prepared on different days). Most explants that failed to show regionally accurate expression were those in which no expression was detected. Few explants (1 of 26 for SCIP and 3 of 54 for NK3) displayed regionally inaccurate expression.

In summary, at E15.5, hippocampal fields appear as multiple, longitudinal, curving bands in the medial wall of the telencephalon. Each band, defined by particular gene expression patterns, has a characteristic width and position. Despite imperfect cell migration within the explants, noted above, multiple bands with remarkably similar widths and positions appear in explants of the E12.5 medial wall maintained for 3 DIV, deprived of all potential extrinsic patterning signals. These observations suggest either (1) a precise regional pattern was already specified in the explants when they were prepared at E12.5, or (2) cues from within the explant itself patterned the tissue during the culture period. Three potential sources of patterning cues within the explants are the Wnt-rich cortical hem (Grove et al., 1998; Lee et al., 2000b), the presumptive extrahippocampal cortex, and tissue interactions within the hippocampal primordium itself. These candidates were evaluated in turn.

\section{Removal of the cortical hem at E12.5 does not disrupt development of field pattern}

The cortical hem is a band of tissue at the extreme medial edge of the cortical neuroepithelium, between the hippocampal primordium and the telencephalic choroid plexus (Grove et al., 1998). The hem expresses multiple Wnt and Bmp genes throughout hippocampal development and may act as a signaling center that directs development of the dorsal telencephalon, including the hippocampus (Furuta et al., 1997; Grove et al., 1998; Grove and Tole, 1999; Lee et al., 2000b).

To determine whether signals from the cortical hem regulate patterning in explants of the E12.5 medial wall, we prepared a new set of explants that lacked the cortical hem (Figs. 1c, 7a). After $3 \mathrm{DIV}$, the explants were processed with two-color in situ hybridization to visualize expression of $W n t 2 b$ and one of three hippocampal markers. Because $W n t 2 b$ expression robustly labels the cortical hem between E11.5 and birth (Grove et al., 1998), absence of $W n t 2 b$ expression in an explant was taken to indicate successful removal of the hem, and only those explants with no cortical hem were used in analysis. Data from four separate culture batches are summarized in Table 2 and illustrated in Figure 7. In most explants, $S C I P, K A 1$, and $N K 3$ are expressed, and marker expression appears in the correct position, given that the most medial part of the tissue has been taken away (Fig. $7 b-d)$. Steel expression was not examined because hem removal was likely to have eliminated the adjacent dentate neuroepithelium as well. Indeed, some explants may have failed to express $N K 3$ or $K A 1$ because medial hippocampal tissue was removed inadvertently.

In mice deficient in Wnt3a, a Wnt gene expressed selectively at the cortical hem, the hippocampus is either absent or represented by tiny, residual cell populations (Lee et al., 2000b). Analysis of Wnt3a-mutant mice at E15.5 or E18.5, for example, reveals no NK3-expressing hippocampal cells and few or no $K A 1$-expressing CA3 cells (Lee et al., 2000b). Wnt3a expression is maintained in the cortical hem in explant culture (data not shown), implying that removing the hem from E12.5 cultures abolishes a Wnt3a signal required for hippocampal field development in vivo. Nonetheless, E12.5 (3 DIV) explants from which the cortical hem was eliminated contain substantial populations of both NK3- and $K A 1$-expressing cells (compare Figs. $5 e, h$ and $7 c, d$ ). These observations suggest that $\mathrm{Wnt} 3 \mathrm{a}$ is most critical to the developing CA fields before E12.5.

\section{Removal of extrahippocampal cortex at E12.5 does not disrupt development of field pattern}

In addition to the hippocampal primordium and the cortical hem, initial E12.5 explants contained portions of presumptive medial neocortex adjacent to the hippocampus, probably including parts of the cingulate and retrosplenial areas. Unlike the cortical hem, this medial cortical tissue has not yet been found to express signaling proteins that might act to pattern the hippocampus. Nonetheless, it has been suggested that this tissue might provide such patterning cues (Tole et al., 1997). To test this possibility, we prepared new E12.5 explants in which presumptive extrahippocampal cortex was dissected away using morphological and 
Figure 7. Manipulating candidate intrinsic patterning cues in E12.5 explants does not disrupt regionspecific marker upregulation. $a$, To determine the effect of the cortical hem on patterning in vitro, E12.5 explants were prepared without the cortical hem (shaded region). To ensure that the hem could be cleanly removed with a single cut, the medial wall was subdivided, and two smaller explants were prepared from each wall. $b-d$, E12.5 (3DIV) explants lacking the hem upregulate marker expression in a spatially appropriate manner. $S C I P$ is expressed laterally (b), and $K A 1$ and $N K 3$ medially (c, d,arrows). In each explant, absence of the cortical hem was confirmed by two-color in situ hybridization in which the regional marker and $W n t 2 b$, a marker of the cortical hem, were identified with different final colors (see Results). Note that dark crystals scattered on and around the edges of the explant represent nonspecific precipitate formed during processing. $e$, To determine the effect of the local environment on marker upregulation, small pieces of the most medial part of the E12.5 medial telencephalic wall were grafted into the lateral part of a host explant. $f, g$, An explant containing a small graft, processed after 3 DIV to show expression of the CA3 marker, KA1.f, White arrowheads indicate the outline of the graft. $K A 1$ expression has been upregulated at the medial edge of both the host explant and the graft $(f, g) . g$, A higher magnification of KA1 expression in the graft. Scale bars: $b-d, 0.55 \mathrm{~mm} ; f, 0.8 \mathrm{~mm} ; g, 0.2 \mathrm{~mm}$.

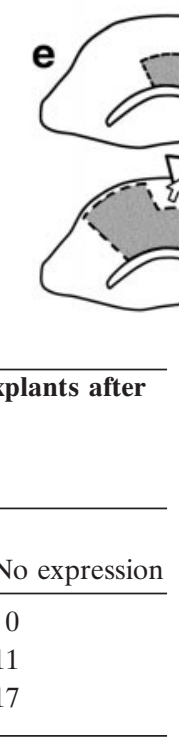

gene expression landmarks described in Materials and Methods. In addition, we prepared some very reduced explants in which almost all of the SCIP-expressing territory was removed, including not only presumptive medial neocortex but also the subicular fields and part of CA1. After 3 DIV, explants were processed as before to show expression of $S C I P, N K 3$, and $K A 1$, each of which was upregulated in a position-appropriate manner (18 of 18 explants, two culture batches), even in the highly reduced explants. Thus, field-specific patterns of gene expression appear in explants deprived of either the cortical hem or the presumptive extrahippocampal cortex. These findings suggest that at E12.5 the hippocampal primordium alone can generate a field pattern.

\section{Expression of a CA3 marker resists modification by cues in a new environment}

Patterning cues during the culture period could also be provided by tissue interactions within the hippocampal primordium itself. To test this possibility, medial fragments of the medial telencephalic wall were grafted into a more lateral position in a larger host explant (Fig. 7e). Thus, presumptive CA3 cells, which can be unequivocally identified by $K A 1$ expression, were confronted with a novel environment of presumptive CA1 and subicular cells. Nonetheless, after 3 DIV, expression of the CA3 marker $K A 1$ was robustly upregulated at an appropriate medial position in each graft (Fig. $7 f, g$ ) (18 of 19 explants, four separate culture batches).

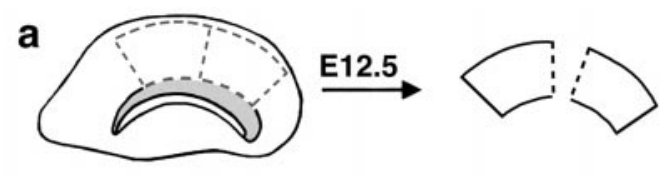

$\begin{array}{lll}\text { SCIP } & \text { KA1 } & \text { NK3 }\end{array}$

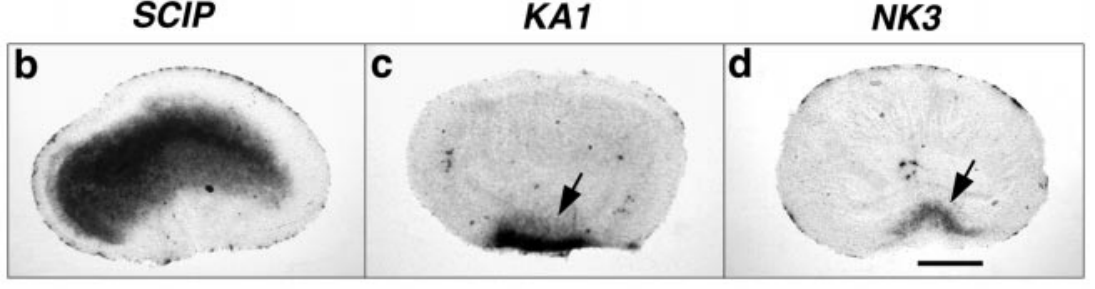

KA1

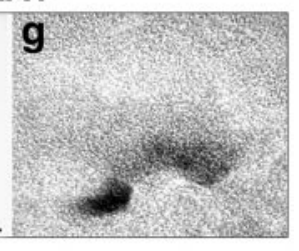

Thus, fragments of the medial wall appear to follow an intrinsic patterning program with respect to $K A 1$ expression. Either the fragments are not responsive to respecification cues in the new, more lateral environment, or no such cues are present.

\section{Explants harvested at the start of hippocampal neurogenesis display intrinsic patterning}

In mouse, the first hippocampal neurons are born at approximately E10.5 (Angevine, 1965; Caviness, 1973; Stanfield and Cowan, 1979). The hippocampal primordium is not morphologically identifiable at this age, but probably lies in the most dorsomedial part of the telencephalon, a region that is in the process of forming the medial walls of each telencephalic hemisphere. Explants of this region were prepared at E10.5 (Fig. 1b) and maintained for 5 DIV to attempt to match the development of the medial telencephalic wall at E15.5. Stringent culture conditions ensured that no undefined factors were available to the explants from extrinsic sources to provide potential patterning cues. Under these conditions, which also provide no extra trophic support to the tissue, E10.5 explants grow less well than those prepared at E12.5, and markers of differentiating neurons, such as class III $\beta$-tubulin, display a reduced, patchy expression (data not shown). Field marker expression appears similarly reduced in E10.5 (5 DIV), compared with E12.5 (3 DIV) explants (Figs. 5b,e,h,k, 8), probably at least partly because of reduced explant health. Nonetheless, the most striking feature of the E10.5 (5 DIV) explants is that regional markers are upregulated with positional accuracy (Fig. 8, Table 3). SCIP is expressed most laterally, NK3 more medially, $K A 1$ more medially still, and Steel near the medial edge of the explant (Fig. 8). Thus, even before a hippocampal primordium is morphologically distinct, some hippocampal patterning information is intrinsic to the dorsomedial telencephalon.

\section{DISCUSSION}

By E12.5, explants of the medial telencephalic wall autonomously generate an accurate array of hippocampal fields with respect to several gene expression patterns. This finding suggests two alter- 
E10.5 (5DIV)

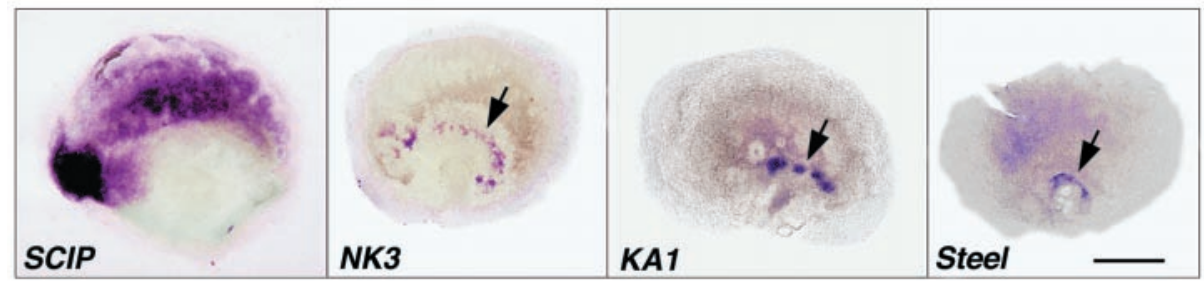

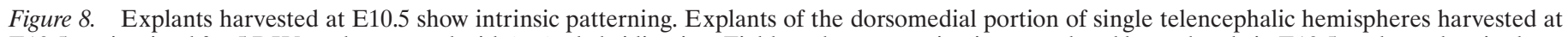

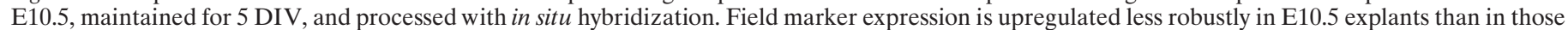

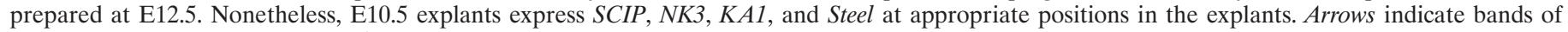
expression of $N K 3, K A 1$, and Steel at different, appropriate lateral-to-medial positions. Scale bar, $0.6 \mathrm{~mm}$.

Table 3. Regional markers upregulated in E10.5 (5DIV) explants

\begin{tabular}{llll} 
& \multicolumn{2}{l}{ \# of explants showing } \\
\cline { 2 - 4 } Marker & $\begin{array}{l}\text { Correct regional } \\
\text { expression }\end{array}$ & $\begin{array}{l}\text { Incorrect regional } \\
\text { expression }\end{array}$ & No expression \\
\hline SCIP & $15(71 \%)$ & 4 & 2 \\
KA1 & $19(66 \%)$ & 4 & 6 \\
NK3 & $15(65 \%)$ & 4 & 4 \\
Steel & $19(63 \%)$ & 10 & 1
\end{tabular}

native hypotheses. Either a field pattern is already specified in the E12.5 medial wall when the explants are placed into culture, or signals from within the explant itself regionalize the tissue during the culture period. The first hypothesis is supported by findings from additional experiments in which candidate sources of intrinsic patterning cues were removed or otherwise manipulated.

These findings are consistent with previous studies (Arimatsu et al., 1992, 1999; Ferri and Levitt, 1993; Cohen-Tannoudji et al., 1994; Eagleson et al., 1997; Levitt et al., 1997; Nothias et al., 1998; Gitton et al., 1999) showing regionalization in the cortical primordium. The new contribution of this study is to examine the relative positioning of several subdivisions within the same broad cortical region. At least with respect to the hippocampus, our observations do not support a view that the area map intrinsic to cortical primordium is a rough sketch that requires considerable refinement from extrinsic cues. Rather, the hippocampal field map at E12.5 appears to be detailed and precise, with well delimited domains already accurately placed with respect to one another.

Previous studies (Eagleson et al., 1997; Arimatsu et al., 1999; Eagleson and Levitt, 1999) show that some region-specific features of cortical cells are specified before the final division of the cell. The present study provides no direct information on whether this is true for the specification of hippocampal cells to express molecular field properties. However, BrdU labeling of E12.5 explants, combined with in situ hybridization to detect neuronand region-specific markers, indicates that a substantial number of cells that go on to differentiate in the explants with respect to these markers are born in the explants. If, as additional findings suggest, the field pattern is already specified in the E12.5 tissue when it is placed into culture, then a reasonable hypothesis is that cells are indeed specified before their final division, which occurs in culture. This issue remains to be explored more directly in future experiments.

An initially surprising observation is how early intrinsic hippocampal patterning is evident. At E10.5, the two telencephalic hemispheres are just forming from a single telencephalic vesicle.
No hippocampal primordium can be distinguished by morphology (Fig. 1), and hippocampal neurogenesis has just begun (Angevine, 1965; Caviness, 1973; Stanfield and Cowan, 1979; present study). Nonetheless, explants of dorsomedial telencephalon harvested at E10.5 upregulate an unmistakable, patterned expression of hippocampal field markers independent of signals from the rest of the brain.

Findings from this study are, however, highly consistent with those of classic morphological and birthdating studies that previously disclosed regional differences in the hippocampus during neurogenesis in both rodents and primates (Caviness, 1973; Nowakowski and Rakic, 1981; Rakic and Nowakowski, 1981; Altman and Bayer, 1990). Notably, in the macaque monkey, the earliest neurons are generated almost simultaneously throughout the hippocampal region, but proliferation ceases at clearly different times for each CA field (Rakic and Nowakowski, 1981). More striking still, and particularly relevant to the findings reported here, an initial "slow" phase of neurogenesis in the macaque hippocampus is followed by a "fast" phase that begins at slightly different times in different fields (Rakic and Nowakowski, 1981). The slightly slower onset of this second phase of neurogenesis in field CA1, compared with neighboring fields, allows the presumptive CA1 region to be transiently distinguished by morphology near the beginning of hippocampal neurogenesis (Nowakowski and Rakic, 1981; Rakic and Nowakowski, 1981). Thus, in the monkey, at least, hippocampal field differences appear close to the onset of neurogenesis. Our own observations, suggesting that a precise molecular field pattern is already specified in the mouse hippocampal primordium at E12.5, are remarkably consistent with these classic observations. Together, these findings indicate the need to identify patterning mechanisms that operate early in hippocampal development.

As is the case for neocortex, cues from extrinsic innervation are available too late. Major extrinsic afferents arrive in the mouse hippocampus between E16.5 and E19.5 (Super and Soriano, 1994). By comparison, molecular field markers are first expressed at E14.5-E15.5 (Tole et al., 1997, 2000a,b; Lee et al., 2000b). The timing of these two events in the mouse is close, suggesting that a small number of afferents that are difficult to detect could invade the embryonic hippocampus before overt fields appear, providing critical patterning cues. However, this study undercuts this possibility by indicating that a field pattern is intrinsic to the hippocampal primordium several days earlier, by E10.5-E12.5. Thus, extrinsic afferents arrive in an already patterned hippocampus in which individual fields could potentially provide cues for precise axon targeting.

The regional identity of cortical cells could, alternatively, be cued by locally released signaling proteins (Ferri and Levitt, 1995; 
Eagleson et al., 1997; Arimatsu et al., 1999; Eagleson and Levitt, 1999; Grove and Tole, 1999; Rubenstein et al., 1999). Findings from the present study suggest that, after E12.5, signals from the cortical hem are not needed for basic CA field patterning. However, the possibility that hem signals might be critical earlier in hippocampal development was not directly tested. Because of the small size of E10.5 explants, the cortical hem could not be removed without potentially removing hippocampal primordial cells as well. Similar considerations prevented testing a later requirement for the cortical hem in the development of the dentate gyrus. Experiments in which the cortical hem is ablated or disrupted in vivo by genetic means could explore these issues more directly.

Meanwhile, previous studies provide evidence that signals from the cortical hem are needed in early hippocampal development. Indirect support for such a role comes from studies of the roofplate in the spinal cord and brainstem, which resembles the cortical hem in dorsal position and expression of Wnt and Bmp genes. Roofplate-derived signals, including those mediated by Wnt and Bmp proteins, regulate the identity and size of dorsal cell groups in the spinal cord and brainstem (Tanabe and Jessell, 1996; Ikeya et al., 1997; Liem et al., 1997; Alder et al., 1999; Lee et al., 2000a; Millonig et al., 2000). Thus, it is plausible that the cortical hem regulates cell identity in the most dorsal part of the developing cortex, the hippocampus. Direct evidence of the importance of Wnt signaling from the cortical hem is supplied by the near-complete loss of the hippocampus in Wnt $3 a$-mutant mice (Lee et al., 2000b) and in mice expressing a mutated form of LEF1 that disrupts Wnt signal transduction by the LEF1/TCF transcription factor family (Galceran et al., 2000). Wnt3a is selectively expressed in the cortical hem before other identified Wnt genes, appearing by E9.75 (Grove et al., 1998; Lee et al., 2000b). Additional Wnt genes are expressed at the cortical hem by E11.5, but this does not rescue hippocampal development in the Wnt3a mutant. These findings suggest that a Wnt signal is critical to hippocampal development before E11.5 and is consistent with the apparent lack of effect of hem removal at E12.5.

Analyses of other mouse lines indicate roles for specific transcription factors in the development of hippocampal subregions. In mice deficient in LEF1, but not in other LEF1/TCF family members, Ammon's horn is grossly normal, but the dentate gyrus is reduced or missing (Galceran et al., 2000). A complementary defect appears in mice with a low dosage of Otx genes, vertebrate homologs of the Drosophila gene orthodenticle. These mice lack Ammon's horn but retain a dentate gyrus (Acampora et al., 1997). These findings suggest a partial dissociation between the dentate gyrus and the CA fields in terms of the mechanisms that regulate their early development. To date, there are few clues to the mechanisms that specify individual CA fields. However, some information is available, in that specification of the CA fields appears not to involve cell lineage (Grove et al., 1992; Martin et al., 1999).

The hippocampus and neocortex are parts of a continuous cortical sheet and show strong similarities in their subcortical connections. Understanding the timing and identity of the mechanisms that pattern the hippocampus may therefore shed light on neocortical area patterning as well. Consistent with this possibility, candidate forebrain signaling centers are positioned to influence both the hippocampus and the neocortex (Grove and Tole, 1999; Rubenstein et al., 1999). Furthermore, transcription factors that may be involved in early cortical regionalization are expressed in patterns that incorporate both the neocortex and the hippocampus (Nakagawa et al., 1999; Liu et al., 2000). Mice deficient in the function of one of these, Emx2, show a patterning defect that affects both types of cortex. In Emx2-mutant mice, the rostral cortex appears expanded (Bishop et al., 2000), whereas caudomedial cortex, including the hippocampus and caudal neocortical areas, is shrunken (Bishop et al., 2000; Mallamaci et al., 2000; Tole et al., 2000a). Such observations reemphasize the continuity of the cortical sheet and indicate that at least some patterning mechanisms may be shared between neocortex and archicortex.

\section{REFERENCES}

Acampora D, Avantaggiato V, Tuorto F, Simeone A (1997) Genetic control of brain morphogenesis through Otx gene dosage requirement. Development 124:3639-3650.

Alder J, Lee KJ, Jessell TM, Hatten ME (1999) Generation of cerebellar granule neurons in vivo by transplantation of BMP-treated neural progenitor cells. Nat Neurosci 2:535-540.

Altman J, Bayer SA (1990) Mosaic organization of the hippocampal neuroepithelium and the multiple germinal sources of dentate granule cells. J Comp Neurol 301:325-342.

Angevine JB (1965) Time of neuron origin in the hippocampal region. Exp Neurol [Suppl]2:1-70.

Arimatsu Y, Miyamoto M, Nihonmatsu I, Hirata K, Uratani Y, Hatanaka Y, Takiguchi-Hayashi K (1992) Early regional specification for a molecular neuronal phenotype in the rat neocortex. Proc Natl Acad Sci USA 89:8879-8883.

Arimatsu Y, Ishida M, Takiguchi-Hayashi K, Uratani Y (1999) Cerebral cortical specification by early potential restriction of progenitor cells and later phenotype control of postmitotic neurons. Development 126:629-638.

Bishop KM, Goudreau G, O'Leary DD (2000) Regulation of area identity in the mammalian neocortex by Emx2 and Pax6. Science 288:344-349.

Caviness VS (1973) Time of neuron origin in the hippocampus and dentate gyrus of normal and reeler mutant mice: an autoradiographic analysis. J Comp Neurol 151:113-120.

Cohen-Tannoudji M, Babinet C, Wassef M (1994) Early determination of a mouse somatosensory cortex marker. Nature 368:460-463.

Donoghue MJ, Rakic P (1999a) Molecular evidence for the early specification of presumptive functional domains in the embryonic primate cerebral cortex. J Neurosci 19:5967-5979.

Donoghue MJ, Rakic P (1999b) Molecular gradients and compartments in the embryonic primate cerebral cortex. Cereb Cortex 9:586-600.

Eagleson KL, Levitt P (1999) Complex signaling responsible for molecular regionalization of the cerebral cortex. Cereb Cortex 9:562-568.

Eagleson KL, Lillien L, Chan AV, Levitt P (1997) Mechanisms specifying area fate in cortex include cell-cycle-dependent decisions and the capacity of progenitors to express phenotype memory. Development 124:1623-1630.

Ferri RT, Levitt P (1993) Cerebral cortical progenitors are fated to produce region-specific neuronal populations. Cereb Cortex 3:187-198.

Ferri RT, Levitt P (1995) Regulation of regional differences in the differentiation of cerebral cortical neurons by EGF family-matrix interactions. Development 121:1151-1160.

Frantz GD, Bohner AP, Akers RM, McConnell SK (1994) Regulation of the POU domain gene SCIP during cerebral cortical development. J Neurosci 14:472-485.

Furuta Y, Piston DW, Hogan BL (1997) Bone morphogenetic proteins (BMPs) as regulators of dorsal forebrain development. Development 124:2203-2212.

Galceran J, Miyashita-Lin EM, Devaney E, Rubenstein JLR, Grosschedl R (2000) Hippocampus development and generation of dentate gyrus granule cells is regulated by LEF1. Development 127:469-482.

Gitton Y, Cohen-Tannoudji M, Wassef M (1999) Specification of somatosensory area identity in cortical explants. J Neurosci 19:4889-4898.

Grove EA, Tole S (1999) Patterning events and specification signals in the developing hippocampus. Cereb Cortex 9:551-561.

Grove EA, Kirkwood TB, Price J (1992) Neuronal precursor cells in the rat hippocampal formation contribute to more than one cytoarchitectonic area. Neuron 8:217-229.

Grove EA, Tole S, Limon J, Yip L, Ragsdale CW (1998) The hem of the embryonic cerebral cortex is defined by the expression of multiple Wnt genes and is compromised in Gli3-deficient mice. Development 125:2315-2325.

Ikeya M, Lee SMK, Johnson JE, McMahon AP, Takada S (1997) Wnt signalling required for expansion of neural crest and CNS progenitors. Nature 389:966-970.

Lee KJ, Dietrich P, Jessell TM (2000a) Genetic ablation reveals that the roof plate is essential for dorsal interneuron specification. Nature 403:734-740. 
Lee SMK, Tole S, Grove EA, McMahon AP (2000b) A local Wnt-3a signal is required for development of the mammalian hippocampus. Development 127:457-467.

Levitt P, Barbe MF, Eagleson KL (1997) Patterning and specification of the cerebral cortex. In: Annual review of neuroscience (Cowan WM, ed), pp 1-24. Palo Alto, CA: Annual Reviews.

Liem Jr KF, Tremml G, Jessell TM (1997) A role for the roof plate and its resident $\mathrm{TGFb}$-related proteins in neuronal patterning in the dorsal spinal cord. Cell 91:127-138.

Liu Q, Dwyer ND, O'Leary DDM (2000) Differential expression of COUP-TFI, CHL1, and two novel genes in developing neocortex identified by differential display PCR. J Neurosci 20:7682-7690.

Mallamaci A, Muzio L, Chan CH, Parnavelas J, Boncinelli E (2000) Area identity shifts in the early cerebral cortex of Emx2-/- mutant mice. Nat Neurosci 3:679-686.

Martin LA, Tan SS, Goldowitz D (1999) Analysis of the role of cell lineage in establishing neural domains in the hippocampal formation using experimental mouse chimeras. Soc Neurosci Abstr 25:503.

Millonig JH, Millen KJ, Hatten ME (2000) The mouse Dreher gene Lmxla controls formation of the roof plate in the vertebrate CNS. Nature 403:764-769.

Miyashita-Lin EM, Hevner R, Wassarman KM, Martinez S, Rubenstein JLR (1999) Early neocortical regionalization in the absence of thalamic innervation. Science 285:906-909.

Motro B, van der Kooy D, Rossant J, Reith A, Bernstein A (1991) Contiguous patterns of c-kit and steel expression: analysis of mutations at the W and Sl loci. Development 113:1207-1221.

Nakagawa Y, Johnson JE, O'Leary DD (1999) Graded and areal expression patterns of regulatory genes and cadherins in embryonic neocortex independent of thalamocortical input. J Neurosci 19:10877-10885.

Nothias F, Fishell G, Ruiz i Altaba A (1998) Cooperation of intrinsic and extrinsic signals in the elaboration of regional identity in the posterior cerebral cortex. Curr Biol 8:459-462.

Nowakowski RS, Rakic P (1981) The site of origin and route and rate of migration of neurons to the hippocampal region of the rhesus monkey. J Comp Neurol 196:129-154.

O'Leary DD (1989) Do cortical areas emerge from a protocortex? Trends Neurosci 12:400-406.

Rakic P (1988) Specification of cerebral cortical areas. Science 241:170-176

Rakic P, Nowakowski RS (1981) The time of origin of neurons in the hippocampal region of the rhesus monkey. J Comp Neurol 196:99-128. Rubenstein JLR, Anderson S, Shi L, Miyashita-Lin E, Bulfone A, Hevner R (1999) Genetic control of cortical regionalization and connectivity. Cereb Cortex 9:524-532.

Shigemoto R, Yokota Y, Tsuchida K, Nakanishi S (1990) Cloning and expression of a rat neuromedin $\mathrm{K}$ receptor cDNA. J Biol Chem 265:623-628.

Stanfield BB, Cowan WM (1979) The development of the hippocampus and dentate gyrus in normal and reeler mice. J Comp Neurol $185: 423-459$

Super H, Soriano E (1994) The organization of the embryonic and early postnatal murine hippocampus. II. Development of entorhinal, commissural, and septal connections studied with the lipophilic tracer DiI. J Comp Neurol 344:101-120.

Tanabe Y, Jessell TM (1996) Diversity and pattern in the developing spinal cord. Science 274:1115-1123.

Tole S, Christian C, Grove EA (1997) Early specification and autonomous development of cortical fields in the mouse hippocampus. Development 124:4959-4970.

Tole S, Assimacopoulos S, Grove EA (2000a) Emx2 is required for growth of the hippocampus but not for hippocampal field specification. J Neurosci 20:2618-2625.

Tole S, Ragsdale CW, Grove EA (2000b) Dorsoventral patterning of the telencephalon is disrupted in the mouse mutant extra-toes $(\mathrm{J})$. Dev Biol 217:254-265.

Wisden W, Seeburg PH (1993) A complex mosaic of high-affinity kainate receptors in rat brain. J Neurosci 13:3582-3598. 